\title{
Changes in peripheral white cell profile and lymphocyte subsets in early onset of anorexia nervosa. The ANABEL Study
}

\author{
A. Gheorghe ${ }^{1}$, P. Andrés ${ }^{2}$, F. Pérez de Heredia ${ }^{1}$, S. Gómez-Martínez ${ }^{1}$, A. Veses ${ }^{1}$, E. Nova ${ }^{1}$, \\ G. Morandé ${ }^{2}$, M. Graell ${ }^{2}$ and A. Marcos ${ }^{1}$ \\ ${ }^{1}$ Immunonutrition Research Group, Dept. Metabolism and Nutrition, Institute of Food Science and Technology and Nutrition \\ ICTAN, Spanish National Research Council CSIC, Madrid, Spain and ${ }^{2}$ Eating Disorders Unit, Dept. Psychiatry, Children \\ University Hospital Niño Jesús, Madrid, Spain
}

Patients with anorexia nervosa (AN) are well known to show altered immune responses; however, results are controversial depending on the evolution period of the illness. Indeed, CD4/CD8 ratio, an index of the nutritional status, has been found to change in the long term ${ }^{(1)}$. Female adolescents ( $n$ 30) diagnosed with AN for up to 12 months were recruited and compared with age- and gender-matched healthy controls (C; $n$ 98). Height and weight values were measured and BMI was calculated. Leucocyte number and profile values were determined (absolute and relative numbers of neutrophils, eosinophils, basophils, monocytes and lymphocytes). Lymphocyte subsets were

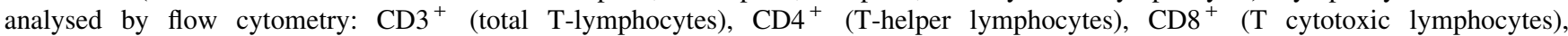
$\mathrm{CD}^{+}{ }^{+} \mathrm{CD} 45 \mathrm{RO}^{+}$and $\mathrm{CD} 8{ }^{+} \mathrm{CD}_{45 \mathrm{RO}^{+}}$(memory T-cells), $\mathrm{CD} 4{ }^{+} \mathrm{CD} 45 \mathrm{RA}^{+}$and $\mathrm{CD} 8{ }^{+} \mathrm{CD} 45 \mathrm{RA}^{+}$(naive T-cells), $\mathrm{CD} 19^{+}$(B-lymphocytes) and natural killer cells $\left(\mathrm{CD} 3^{-} \mathrm{CD} 16^{+} 56^{+}\right)$. Total leucocytes, neutrophils, basophils and monocytes were lower in early AN patients than in $\mathrm{C}(P<0.05)$, and so were natural killer and memory $\left(\mathrm{CD} 4{ }^{+} \mathrm{CD} 45 \mathrm{RO}^{+}\right.$and $\left.\mathrm{CD} 8{ }^{+} \mathrm{CD} 45 \mathrm{RO}{ }^{+}\right)$cells $(P<0.001)$. Total $\mathrm{CD}^{+} \mathrm{T}$-cell number was also lower $(P<0.05)$ in $\mathrm{AN}$ patients than in $\mathrm{C}$, but $\mathrm{CD}^{+}{ }^{+}$remained similar in both groups, leading to a higher CD4/CD8 ratio in AN patients $(P=0.001)$. These results show a modified behaviour of immunocompetent cells in AN. The leucocyte profile and lymphocyte subset values suggest that AN patients might develop a reduced response capacity against antigen challenges, despite showing unmodified humoral immunity (measured as CD19+ ${ }^{+}$counts) and a higher CD4/CD8 ratio compared with controls.

\begin{tabular}{|c|c|c|c|c|c|c|c|c|c|c|}
\hline & \multicolumn{5}{|c|}{ Relative values (\%) } & \multicolumn{5}{|c|}{ Absolute values (cells/ml) } \\
\hline & \multicolumn{2}{|c|}{ Controls } & \multicolumn{2}{|c|}{ AN patients } & \multirow[b]{2}{*}{$P$} & \multicolumn{2}{|c|}{ Controls } & \multicolumn{2}{|c|}{ AN patients } & \multirow[b]{2}{*}{$P$} \\
\hline & Mean & SD & Mean & $\mathrm{SD}$ & & Mean & SD & Mean & SD & \\
\hline $\mathrm{CD}_{19}^{+}$ & 11.1 & 2.89 & 12.4 & 4.21 & NS & 251 & 89.0 & 264 & 106.7 & NS \\
\hline $\mathrm{CD}^{-}{ }^{-} \mathrm{CD} 16^{+} 56^{+}$ & 16.5 & 6.57 & 9.6 & 4.46 & $<0.001$ & 382 & 196.4 & 206 & 104.2 & $<0.001$ \\
\hline $\mathrm{CD}^{+}{ }^{+} \mathrm{CD} 45 \mathrm{RO}^{+}$ & 19.6 & 9.99 & 38.5 & 15.11 & $<0.001$ & 750 & 249.3 & 887 & 260.2 & 0.012 \\
\hline $\mathrm{CD}^{+}$ & 27. & 5.64 & 24.0 & 4.73 & 0.010 & 651 & 212.0 & 513 & 145.4 & 0.015 \\
\hline $\mathrm{CD}^{+} \mathrm{CD}^{+} 5 \mathrm{RO}^{+}$ & 42.0 & 13.64 & 24.2 & 11.71 & $<0.001$ & 242 & 127.7 & 139 & 59.4 & $<0.001$ \\
\hline $\mathrm{CD}^{+} \mathrm{CD}^{+} 5 \mathrm{RA}^{+}$ & 58.2 & 3.02 & 60.9 & 21.23 & NS & 330 & 145.0 & 341 & 120.4 & NS \\
\hline $\mathrm{CD}^{+}{ }^{+}$ & 37.7 & 6.55 & 42.0 & 7.10 & 0.002 & 861 & 281.6 & 905 & 276.9 & NS \\
\hline $\mathrm{CD}^{+}{ }^{+} \mathrm{CD}_{45 \mathrm{RO}}{ }^{+}$ & 51.6 & 10.92 & 24.6 & 7.92 & $<0.001$ & 406 & 161.3 & 238 & 76.1 & $<0.001$ \\
\hline $\mathrm{CD}^{+}{ }^{+} \mathrm{CD} 4 \mathrm{RA}{ }^{+}$ & 48.1 & 10.71 & 44.2 & 18.07 & NS & 388 & 177.2 & 456 & 212.0 & NS \\
\hline CD4/CD8 & & - & & - & & 1.47 & 0.466 & 1.82 & 0.506 & 0.001 \\
\hline
\end{tabular}

Values are presented as means and SD. Differences between means were analysed by Student's $t$ test and considered significant at $P<0.05$ 